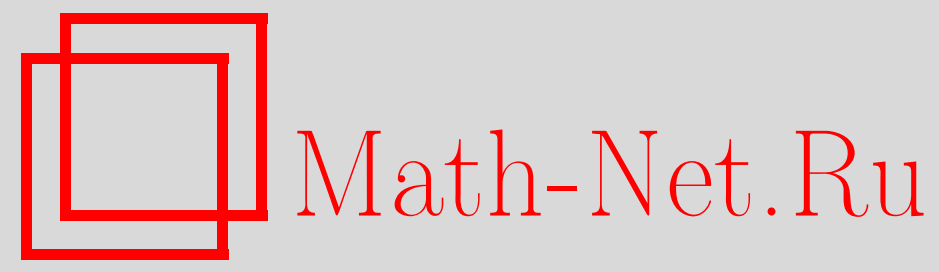

Р. Ш. Липцер, Большие уклонения для эмпирических мер марковских процессов (дискретное время, некомпактный случай), Теория вероятн. и ее примен., 1996, том 41, выпуск 1, 65-88

DOI: https://doi.org/10.4213/tvp2776

Использование Общероссийского математического портала Math-Net.Ru подразумевает, что вы прочитали и согласны с пользовательским соглашением http://www.mathnet.ru/rus/agreement

Параметры загрузки:

IP : 54.174 .149 .18

26 апреля 2023 г., $12: 16: 18$

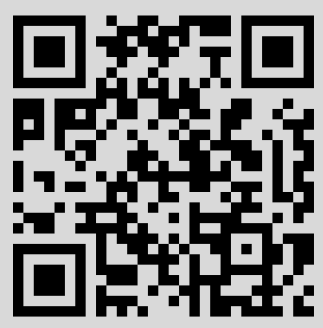




\title{
БОЛЬШИЕ УКЛОНЕНИЯ ДЛЯ ЭМПИРИЧЕСКИХ МЕР МАРКОВСКИХ ПРОЩЕССОВ (дискретное время, некомпактный случай) ${ }^{1)}$
}

\begin{abstract}
Приводится простое доказательство принципа больших уклонений Донскера и Варадана для семейства эмпирических мер марковского процесса с дискретным временем со значениями в $\mathbf{R}$ (не компактный случай). Доказательство не опирается ни на понятие условной энтропии, ни на результаты так называемого «третьего уровня» в больших уклонениях. Оно основано на теореме Пухальского и представляет собой новый вариант доказательства принципа больших уклонений Дюпуи и Эллиса для двумерных эмпирических мер, что позволяет заменить предположение о существовании инвариантной меры более естественным с точки зрения приложений условием. Приводится пример марковского процесса, задаваемого нелинейным рекуррентным соотношением, для которого условия сушествования принципа больших уклонений проверяются.
\end{abstract}

Клочевые слова и фразы: большие уклонения, экспоненциальная плотность, локальный принцип больших уклонений.

\section{1. Введение. Формулировка основных результатов}

1. Рассмотрим однородный марковский процесс $\xi=\left(\xi_{k}\right)_{k \geqslant 0}$, принимающий значения в $\mathbf{R}$, с начальным распределением $\alpha_{0}(d x)$. При фиксированной начальной точке $\xi_{0}=x$ Донскер и Варадан [1] установили принцип больших уклонений (п.б.у.) для семейства $\left(\pi_{n}, n \geqslant 1\right)$ эмпирических распределений (оккупащионных мер):

$$
\pi_{n}(A)=\frac{1}{n} \sum_{k=1}^{n} I\left(\xi_{k} \in A\right),
$$

при выполнении следующих предположений: ской;

(F) переходная вероятность $\pi(x, d y)$ процесса $\xi$ является феллеров-

*Tel Aviv University, Dept. of Electrical Engineering-Systems, 69978 Tel Aviv, Israel.

1) Эта работа выполнена при финансовой поддержке Российского фонда фундаментальных исследований \# 93-011-1450.

3 Теория вероятностей и ее применения, № 1 
(I) существует единственная инвариантная мера $\alpha(d x)$ :

$$
\alpha(\Gamma)=\int_{\mathbf{R}} \pi(x, \Gamma) \alpha(d x), \quad \Gamma \in \mathbf{R}
$$

$\left(\mathbf{H}^{*}\right)$ найдется неотрицательная измеримая функция $v(x)$ такая, что $\sup _{|x| \leqslant N} v(x)<\infty$ для любого $N>0$, и для функции

$$
w(x)=\ln \frac{e^{v(x)}}{\int_{\mathbf{R}} e^{v(y)} \pi(x, d y)}
$$

выполняются свойства inf $x \in \mathbf{R} w(x)=w_{*}>-\infty, \lim _{i \rightarrow \infty} \inf _{|x|>i}[w(x)-$ $\left.w_{*}\right]=\infty$;

$(\mathbf{R M})$ сушествует $\sigma$-конечная мера $l=l(d x)$ такая, что

$$
\pi(x, d y)=p(y \mid x) l(d x) \quad \alpha-\Pi . \text { н. }
$$

и для каждого $x$

$$
p(y \mid x)>0 \quad l \text {-п.н. }
$$

Для точной формулировки результата Донскера и Варадана введем метрическое пространство (S, $\rho)$ (S - множество вероятностных мер на $\mathbf{R}, \rho$ - метрика Леви-Прохорова).

Теорема (Донскер, Варадан). Если выполнены условия (F), (I) $\left(\mathbf{H}^{*}\right) u(\mathbf{R M}), m o$

(i) для любого замкнутого (в метрике $\rho$ ) множества $F$

$$
\limsup _{n} \frac{1}{n} \ln \mathbf{P}\left\{\pi_{n} \in F\right\} \leqslant-\inf _{\mu \in F} J(\mu),
$$

(ii) для любого открытого (в метрике $\rho$ ) множества $G$

$$
\liminf _{n} \frac{1}{n} \ln \mathbf{P}\left\{\pi_{n} \in G\right\} \geqslant-\inf _{\mu \in G} J(\mu),
$$

где $J(\mu)$ - бункиионал действия, определяемый формулой

$$
J(\mu)=\sup _{u \in \mathfrak{N}} \int_{\mathbf{R}} \ln \frac{e^{u(x)}}{\int_{\mathbf{R}} e^{u(y)} \pi(x, d y)} \mu(d x),
$$

$u \mathfrak{N}$ - множество непрерывных финитных функиий.

2. На протяжении всей работы будут использоваться обозначения:

$$
\lambda_{\gamma}^{\circ}(d x, d y)=\pi(x, d y) \gamma(d x), \quad \forall \gamma \in \mathbf{S},
$$

и $\mathbf{S}_{\gamma \gamma}$ для множества вероятностных мер на $\mathbf{R}^{2}$ с одинаковыми маргиналами $\gamma$. Для $\lambda \in \mathbf{S}_{\mu \mu}$ количество

$$
H\left(\lambda \mid \lambda_{\mu}^{\circ}\right)= \begin{cases}\int_{\mathbf{R}^{2}} \ln \frac{d \lambda}{d \lambda_{\mu}^{\circ}}(x, y) \lambda(d x, d y), & \lambda \ll \lambda_{\mu}^{\circ}, \\ \infty, & \text { в остальных случаях, }\end{cases}
$$


будем называть, следуя Донскеру и Варадану, условной энтропией меры $\lambda$ относительно меры $\lambda_{\mu}^{\circ}$.

Существенным моментом в доказательстве Донскера и Варадана в части, касающейся нижней оценки, является тождество

$$
J(\mu) \equiv \inf _{\lambda \in \mathbf{S}_{\mu \mu}} H\left(\lambda \mid \lambda_{\mu}^{\circ}\right) \quad\left(=J^{\prime}(\mu)\right)
$$

Следует отметить, что неравенство $J(\mu) \leqslant J^{\prime}(\mu)$ является очевидным, в то время как доказательство неравенства $J(\mu) \geqslant J^{\prime}(\mu)$ даже в компактном случае (см. [2]) выглядит местами весьма сложно. Позднее Донскер и Варадан установили п.б.у. не прибегая к тождеству $J(\mu) \equiv J^{\prime}(\mu)$. Предложенный ими подход использует метод непрерывных отображений (так называемый «contraction principle» Варадана [3]) и п.б.у. для оккупационных полей (эмпирических мер «третьего уровня» [4]).

3. Цель работы - установить п.б.у. Донскера и Варадана и его обобшения, не используя ни «третий уровень», ни тождество $J(\mu) \equiv$ $J^{\prime}(\mu)$.

В настоящей работе также не предполагается существование инвариантной меры у переходной вероятности $\pi(x, d y)$. Поэтому вместо условий (I) и (RM) вводятся условия:

$\left(\mathrm{I}^{\prime}\right)$ найдется хотя бы одна вероятностная мера $\lambda^{\prime}=\lambda^{\prime}(d x, d y)$ на $\mathbf{R}^{2}$ с одинаковыми маргиналами, обозначаемыми $\alpha=\alpha(d x)$, такая, что

1) $\lambda^{\prime} \sim \lambda_{\alpha}^{\circ}$

2) $H\left(\lambda^{\prime} \mid \lambda_{\alpha}^{o}\right)<\infty$

((I) влечет за собой $\left(\mathbf{I}^{\prime}\right)$ с $\left.\lambda^{\prime}=\lambda_{\alpha}^{\circ}\right)$;

$\left(\mathbf{R M}^{\prime}\right)$ у переходных вероятностей $\pi(x, d y)$ и $\pi^{\prime}(x, d y)\left(\lambda^{\prime}(d x, d y)=\right.$ $\left.\pi^{\prime}(x, d y) \alpha(d x)\right)$ существуют условные плотности $p(y \mid x)$ и $p^{\prime}(y \mid x)$ относительно некоторой $\sigma$-конечной меры $l=l(d x)$ такие, что при каждом $x \in \mathbf{R}$

$$
\begin{aligned}
\pi(x, d y) & =p(y \mid x) l(d y), \\
\pi^{\prime}(x, d y) & =p^{\prime}(y \mid x) l(d y) \quad \alpha \text {-п.н. }
\end{aligned}
$$

и $p^{\prime}(y \mid x)>0(l \times \alpha)$-п.н.

С начальной мерой $\alpha_{0}$ свяжем еше одно условие: что

$\left(\mathbf{H}_{0}\right)$ функция $u(x)=v(x)+\frac{1}{2}\left[w(x)-w_{*}\right]$, где $v(x)$ из $\left(\mathbf{H}^{*}\right)$, такова,

$$
\exists b>0: \int_{\mathbf{R}} e^{b u(x)} \alpha_{0}(d x)<\infty
$$

и

$$
\int_{\mathbf{R}} p(y \mid x) \alpha_{0}(d x)>0 \quad l \text {-П.н. }
$$

Основной результат приводится в нижеследующей теореме.

Теорема 1.1. Пусть выполнены условия $(\mathbf{F}),\left(\mathbf{I}^{\prime}\right),\left(\mathbf{H}^{*}\right),\left(\mathbf{R} \mathbf{M}^{\prime}\right)$ u $\left(\mathbf{H}_{0}\right)$. Тогда семейство $\left(\pi_{n}, n \geqslant 1\right)$ удовлетворяет п.б.у. в $(\mathbf{S}, \rho)$ c 
Функчионалом действия

$$
J^{\prime}(\mu)=\inf _{\lambda \in \mathbf{S}_{\mu \mu}} H\left(\lambda \mid \lambda_{\mu}^{\circ}\right), \quad \forall \mu \in \mathbf{S} .
$$

Для любых замкнутого $F$ и открытого $G$ (в метрике $\rho$ ) множеств

$$
\begin{aligned}
& \underset{n}{\limsup } \frac{1}{n} \ln \mathbf{P}\left\{\pi_{n} \in F\right\} \leqslant-\inf _{\mu \in F} J^{\prime}(\mu), \\
& \liminf _{n} \frac{1}{n} \ln \mathbf{P}\left\{\pi_{n} \in G\right\} \geqslant-\inf _{\mu \in G} J^{\prime}(\mu) .
\end{aligned}
$$

Для доказательства этой теоремы используется метод непрерывных отображений («contraction principle») и п.б.у. для эмпрических мер $\pi_{n}^{2}(d x, d y)$ :

$$
\pi_{n}^{2}(A \times B)=\frac{1}{n} \sum_{k=1}^{n} I\left(\xi_{k-1} \in A, \xi_{k} \in B\right)
$$

(см. [5], [6]).

4. Введем метрическое пространство $\left(\mathbf{S}^{2}, \rho^{2}\right)$, где $\mathbf{S}^{2}-$ пространство вероятностных мер на $\mathbf{R}^{2}$ и $\rho^{2}$ - метрика Леви-Прохорова. П.б.у. для семейства $\left(\pi_{n}^{2}, n \geqslant 1\right)$ дает следуюшая теорема.

Теорема 1.2. Пусть выполнень условия $(\mathbf{F}),\left(\mathbf{I}^{\prime}\right),\left(\mathbf{H}^{*}\right),\left(\mathbf{R M}^{\prime}\right) u$ $\left(\mathbf{H}_{0}\right)$. Тодда семейство $\left(\pi_{n}^{2}, n \geqslant 1\right)$ удовлетворяет п.б.y. в $\left(\mathbf{S}^{2}, \rho^{2}\right) c$ функиионалом действия $J^{2}=J^{2}(\lambda), \lambda \in \mathbf{S}^{2}, c$

$$
J^{2}(\lambda)=\left\{\begin{array}{llll}
\infty, & \text { маргиналы } & \lambda & \text { различны, } \\
H\left(\lambda \mid \lambda_{\gamma}^{\circ}\right), & \text { мараиналы } & \lambda & \text { совпадают }(=\gamma) .
\end{array}\right.
$$

Для любых замкнутого $F^{2}$ и открытого $G^{2}$ (в метрике $\left.\rho^{2}\right)$ множеств

$$
\begin{gathered}
\limsup _{n} \frac{1}{n} \ln \mathbf{P}\left\{\pi_{n}^{2} \in F^{2}\right\} \leqslant-\inf _{\lambda \in F^{2}} J^{2}(\lambda), \\
\liminf _{n} \frac{1}{n} \ln \mathbf{P}\left\{\pi_{n}^{2} \in G^{2}\right\} \geqslant-\inf _{\lambda \in G^{2}} J^{2}(\lambda) .
\end{gathered}
$$

В последнем разделе работы приводится пример марковского процесса, для которого проверка условий теорем 1.1 и 1.2 эффективна.

5. Теорема 1.2 несколько отличается по формулировке и существенно по методу доказательства от [5] и [6]. Чтобы пояснить это, нам. потребуются следующие понятия.

О п р е д е л е н и е 1 [7]. Семейство $\left(\pi_{n}^{2}, n \geqslant 1\right)$ является әкспоненциально плотным в $\left(\mathbf{S}^{2}, \rho^{2}\right)$, если найдется последовательность компактов $K_{j}^{2} \in \mathbf{S}^{2}, j \geqslant 1$, такая, что.

$$
\underset{n}{\limsup } \frac{1}{n} \ln \mathbf{P}\left\{\pi_{n}^{2} \in \mathbf{S}^{2} \backslash K_{j}^{2}\right\}=-\infty .
$$


О п р е д е л е н и е 2 [8]. Семейство $\left(\pi_{n}^{2}, n \geqslant 1\right)$ удовлетворяет частичному принчипу больших уклонений в $\left(\mathbf{S}^{2}, \rho^{2}\right)$, если всякая бесконечная подпоследовательность $\left(\pi_{n^{\prime}}^{2}\right)$ последовательности $\left(\pi_{n}^{2}\right)$ содержит подпоследовательность $\left(\pi_{\tilde{n}}^{2}\right)$, удовлетворяющую п.б.у. в $\left(\mathbf{S}^{2}, \rho^{2}\right)$ с некоторым функционалом действия $\tilde{J}^{2}=\tilde{J}^{2}(\lambda)$.

O п р е д е л е н и е 3 (ср. с [9]). Семейство $\left(\pi_{n}^{2}, n \geqslant 1\right)$ удовлетворяет локальному п.6.y. в $\left(\mathbf{S}^{2}, \rho^{2}\right)$, если для любого $\lambda \in \mathbf{S}^{2}$

$$
\begin{aligned}
& \liminf _{\delta \rightarrow 0} \liminf _{n} \frac{1}{n} \ln \mathbf{P}\left\{\rho^{2}\left(\pi_{n}^{2}, \lambda\right) \leqslant \delta\right\} \\
& \quad=\limsup _{\delta \rightarrow 0} \limsup _{n} \frac{1}{n} \ln \mathbf{P}\left\{\rho^{2}\left(\pi_{n}^{2}, \lambda\right) \leqslant \delta\right\} \quad(=-I(\lambda)) .
\end{aligned}
$$

В теореме 2.1 при условиях $\left(\mathbf{H}^{*}\right)$ и $\left(\mathrm{H}_{0}\right)$ устанавливается экспоненциальная плотность семейства $\left(\pi_{n}^{2}, n \geqslant 1\right)$. Комбинация теорем 3.1 и 4.1 дает локальный п.б.у. для этого семейства с

$$
I(\lambda) \equiv J^{2}(\lambda) .
$$

Связуюшим звеном при доказательстве теоремы 1.2 служит теорема Пухальского [8], которую удобно представить здесь в виде импликации:

$$
\text { «экспоненщиальная плотность» } \Longleftrightarrow \text { «частичный п.б.у.». }
$$

Итак, схема доказательства теоремы 1.2 выглядит следуюшим образом.

По теореме $2.1\left(\pi_{n}^{2}, n \geqslant 1\right)$ - экспоненциально плотное семейство. По теореме Пухальского можно взять подпоследовательность $\left(\pi_{\tilde{n}}^{2}\right)$, удовлетворяюпую п.б.у. с функционалом действия $\tilde{J}^{2}(\lambda)$, являюшимся полунепрерывной снизу функцией. Эти факты влекут за собой локальный п.б.у. для $\left(\pi_{\widetilde{n}}^{2}\right)$ : для любого $\lambda \in \mathbf{S}^{2}$

$$
\begin{aligned}
& \liminf _{\delta \rightarrow 0} \liminf _{\widetilde{n}} \frac{1}{\widetilde{n}} \ln \mathbf{P}\left\{\rho^{2}\left(\pi_{\tilde{n}}^{2}, \lambda\right) \leqslant \delta\right\} \\
& \quad=\limsup _{\delta \rightarrow 0} \lim \sup _{\tilde{n}} \frac{1}{\widetilde{n}} \ln \mathbf{P}\left\{\rho^{2}\left(\pi_{\tilde{n}}^{2}, \lambda \leqslant \delta\right\} \quad\left(=-\tilde{J}^{2}(\lambda)\right) .\right.
\end{aligned}
$$

Очевидное нера́венство

$$
\liminf _{n} \leqslant \liminf _{\widetilde{n}} \leqslant \limsup _{\widetilde{n}} \leqslant \underset{n}{\limsup }
$$

и $(1.7),(1.8),(1.10)$ приводят к тождеству

$$
\tilde{J}^{2}(\lambda) \equiv J^{2}(\lambda)
$$

т.е. $J^{2}(\lambda)$ - функционал действия (этот же прием использовался в $[10]$ ). Верхние и нижние оценки теоремы 1.2 устанавливаются также с привлечением теоремы Пухальского. 
По поводу других подходов к установлению п.б.у. для семейств $\left(\pi_{n}\right)$ и $\left(\pi_{n}^{2}\right)$ см. [11]-[14].

6. Как отмечалось выше, равенство (1.8) вытекает из теорем 3.1 и 4.1 для верхних и нижних оџенок локального п.б.у. семейства $\left(\pi_{n}^{2}\right.$, $n \geqslant 1)$. Доказательство нижней оценки требует лишь условий $\left(\mathbf{I}^{\prime}\right)$ и $\left(\mathbf{R} \mathbf{M}^{\prime}\right)$ и использует стандартный прием локально абсолютно непрерывной замены меры, предложенный в работах Донскера и Варадана [1], [2], и идею регуляризации из недавней работы Ву [15]. По этому же поводу следует отметить работу Джейна [16].

Доказательство верхней оценки локального п.б.у. семейства $\left(\pi_{n}^{2}\right.$, $n \geqslant 1)$ требует свойства феллеровости $(\mathbf{F})$ и опирается на экспоненциальную плотность этого семейства.

\section{2. Экспоненциальная плотность семейства $\left(\pi_{n}^{2}, n \geqslant 1\right)$}

В соответствии с определением 1 достаточно проверить (1.6). Чтобы сделать это, нам потребуется ряд вспомогательных результатов.

Возьмем положительную убывающую функцию $\gamma=\gamma(y), y \geqslant 0$, такую, что $\lim _{y \rightarrow \infty} \gamma(y)=0$, и положим

$$
K_{j}^{2}=\bigcap_{i \geqslant j}\left\{\lambda \in \mathbf{S}^{2}: \int_{(|x|>i) \cup(|y|>i)} \lambda(d x, d y) \leqslant \gamma(i)\right\} .
$$

Множество $K_{j}^{2}$ является плотным и, в силу теоремы Прохорова (см. $[17])$, относительно компактным. Поскольку $\{x, y:(|x|>l) \cup(|y|>l)\}-$ открытое множество, предел любой сходящейся последовательности из $K_{j}^{2}$ принадлежит $K_{j}^{2}$, т.е. $K_{j}^{2}-$ компакт. Очевидно, что $K_{j}^{2} \subseteq K_{j+1}^{2}$.

Для $j \geqslant 1$ и $\lambda \in \mathbf{S}^{2}$ положим

$$
L(j, \lambda)=\min \left\{i \geqslant j: \int_{(|x|>i) \cup(|y|>i)} \lambda(d x, d y)>\gamma(i)\right\} .
$$

Лемма 2.1. Семейство ( $\left.\pi_{n}^{2}, n \geqslant 1\right)$ әкспоненциально плотно в $\left.\left(\mathbf{S}^{2}, \rho^{2}\right), e c\right\lrcorner u$

$$
\lim _{j} \limsup _{n} \frac{1}{n} \ln \mathbf{P}\left\{L\left(j, \pi_{n}^{2}\right)<\infty\right\}=-\infty .
$$

Д ок а 3 а те ль с т в о. Возьмем компакт $K_{j}^{2}$, определенный в (2.1), и заметим, что

$$
\left\{\pi_{n}^{2} \in \mathbf{S}^{2} \backslash K_{j}^{2}\right\}=\left\{L^{2}\left(j, \pi_{n}\right)<\infty\right\} .
$$

Тогда (1.6) является следствием (2.3).

Лемма 2.2. Для любых измеримых множеств $A_{n}, n \geqslant 1, u B_{n, i}$, $n \geqslant 1, i \geqslant 1$, со свойством $\lim _{i \rightarrow \infty} \lim \sup _{n}(1 / n) \ln \mathbf{P}\left(B_{n, i}\right)=-\infty$ имеет 
место равенство

$$
\underset{n}{\limsup } \frac{1}{n} \ln \mathbf{P}\left(A_{n}\right)=\underset{i \rightarrow \infty}{\limsup } \limsup _{n} \frac{1}{n} \ln \mathbf{P}\left(A_{n}, \Omega \backslash B_{n, i}\right) .
$$

Доказ ат ель с т в о. Требуемый результат вытекает из очевидных неравенств $\mathbf{P}\left(A_{n}\right) \geqslant \mathbf{P}\left(A_{n}, \Omega \backslash B_{n, i}\right) ; \mathbf{P}\left(A_{n}\right) \leqslant 2\left[\mathbf{P}\left(A_{n}, \Omega \backslash B_{n, i}\right) \vee\right.$ $\left.\mathbf{P}\left(B_{n, i}\right)\right]$.

Теорема 2.1. Пусть выполнены условия $\left(\mathbf{H}^{*}\right)$ u $\left(\mathbf{H}_{0}\right)$. Тогда семейство $\left(\pi_{n}^{2}, n \geqslant 1\right)$ экспоненциально плотно в $\left(\mathbf{S}^{2}, \rho^{2}\right)$.

Д о к а з а те л в с т в о. Возьмем

$$
\gamma(y)=\frac{1}{\sqrt{\inf _{|x|>y}\left[w(x)-w_{*}\right]}}
$$

где $w(x)$ - функция из условия $\left(\mathrm{H}^{*}\right)$, и покажем, что соотношение $(2.3)$ выполняется с этой функцией $\gamma(y)$.

В силу условия $\left(\mathbf{H}_{0}\right)$ и неравенства Чебышева

$$
\mathbf{P}\left\{u\left(\xi_{0}\right)>i n\right\} \leqslant e^{-(i n) b} \int_{\mathbf{R}} e^{b u(x)} \alpha_{0}(d x) .
$$

Следовательно, для $B_{n, i}=\left\{v\left(\xi_{0}\right) \geq i n\right\}$

$$
\limsup _{n} \frac{1}{n} \ln \mathbf{P}\left(B_{n, i}\right) \leqslant-i b \rightarrow-\infty, \quad i \rightarrow \infty .
$$

Поэтому в силу лемм 2.1 и 2.2 экспоненциальная плотность семейства $\left(\pi_{n}^{2}, n \geqslant 1\right)$ будет иметь место, если

$\lim _{i} \limsup _{j} \limsup \frac{1}{n} \ln \mathbf{P}\left\{L\left(j, \pi_{n}^{2}\right)<\infty, v\left(\xi_{0}\right) \leqslant i n\right\}=-\infty$.

Для того, чтобы проверить (2.5), воспользуемся включениями (напомним, что $\left.L\left(j, \pi_{n}^{2}\right) \geqslant j\right)$ :

$$
\begin{aligned}
& \left\{L\left(j, \pi_{n}^{2}\right)<\infty\right\} \subseteq\left\{\int_{\left(|x|>L\left(j, \pi_{n}^{2}\right)\right) \cup\left(|y|>L\left(j, \pi_{n}^{2}\right)\right)} \pi_{n}^{2}(d x, d y)>\gamma\left(L\left(j, \pi_{n}^{2}\right)\right)\right\} \\
& \subseteq\left\{\int_{|x|>L\left(j, \pi_{n}^{2}\right)} \int_{\mathbf{R}} \pi_{n}^{2}(d x, d y)+\int_{\mathbf{R}} \int_{|y|>L\left(j, \pi_{n}^{2}\right)} \pi_{n}^{2}(d x, d y)>\gamma\left(L\left(j, \pi_{n}^{2}\right)\right)\right\} \\
& \subseteq\left\{2 \int_{|x|>L\left(j, \pi_{n}^{2}\right)} \int_{\mathbf{R}} \pi_{n}(d x)+\frac{1}{n} I\left(\left|\xi_{0}\right|>L\left(j, \pi_{n}^{2}\right)\right)>\gamma\left(L\left(j, \pi_{n}^{2}\right)\right)\right\} .
\end{aligned}
$$

Тогда (2.5) выполняется при условии, что

$$
\begin{aligned}
& \underset{j}{\limsup } \underset{n}{\limsup } \frac{1}{n} \ln \mathbf{P}\left\{\int_{|x|>j} \pi_{n}(d x)+\frac{1}{2 n} I\left(\left|\xi_{0}\right|>L\left(j, \pi_{n}^{2}\right)\right)\right. \\
& \left.>\gamma\left(L\left(j, \pi_{n}^{2}\right)\right), u\left(\xi_{0}\right) \leqslant i n\right\}=-\infty
\end{aligned}
$$


Положим $\left.Z_{n}=\prod_{k=0}^{n-1}\left[e^{v\left(\xi_{k+1}\right)} / \mathbf{E} e^{v\left(\xi_{k+1}\right)} \mid \xi_{k}\right)\right]$, где $v(x)$ - функция из условия $\left(\mathbf{H}^{*}\right)$. В силу марковского свойства:

$$
\mathbf{E}\left(e^{v\left(\xi_{k+1}\right)}, \mid \xi_{k}\right)=\mathbf{E}\left(e^{v\left(\xi_{k+1}\right)} \mid \xi_{0}, \ldots, \xi_{k}\right) \quad \mathbf{P} \text {-п.н. }
$$

и, значит, имеют место равенство $\mathrm{E} Z_{n}=1$ и очевидное неравенство

$$
\begin{aligned}
1 \geqslant \operatorname{E} I\left(\int_{|x|>L\left(j, \pi_{n}^{2}\right)}\right. & \pi_{n}(d x)+\frac{1}{2 n} I\left(\left|\xi_{0}\right|>L\left(j, \pi_{n}^{2}\right)\right) \\
& \left.>\gamma\left(L\left(j, \pi_{n}^{2}\right)\right), u\left(\xi_{0}\right) \leqslant i n\right) Z_{n} .
\end{aligned}
$$

Из определения $Z_{n}$ и $w(x)\left(\right.$ см. $\left.\left(\mathrm{H}^{*}\right)\right)$ следует, что

$$
\begin{aligned}
\ln Z_{n} & =\sum_{k=1}^{n} v\left(\xi_{k}\right)-\sum_{k=1}^{n} \ln \int_{\mathbf{R}} e^{v(y)} \pi\left(\xi_{k-1}, d y\right)=v\left(\xi_{n}\right)-v\left(\xi_{0}\right)+\sum_{k=1}^{n} w\left(\xi_{k-1}\right) \\
& =v\left(\xi_{n}\right)-v\left(\xi_{0}\right)+n w_{*}+n \int_{\mathbf{R}}\left[w(x)-w_{*}\right] \pi_{n}(d x) .
\end{aligned}
$$

Оценим снизу $\ln Z_{n}$ на множестве

$$
\left\{\int_{|x|>L\left(j, \pi_{n}^{2}\right)} \pi_{n}(d x)+\frac{1}{2 n} I\left(\left|\xi_{0}\right|>L\left(j, \pi_{n}^{2}\right)\right)>\gamma\left(L\left(j, \pi_{n}^{2}\right)\right)\right\} .
$$

Имеем (напомним, что $L\left(j, \pi_{n}^{2}\right) \geqslant j$ ):

$$
\begin{aligned}
\ln Z_{n} \geqslant & -v\left(\xi_{0}\right)+n w_{*}+n \inf _{|y|>L\left(j, \pi_{n}^{2}\right)}\left[w(x)-w_{*}\right] \frac{1}{2} \gamma\left(L\left(j, \pi_{n}^{2}\right)\right) \\
& -\frac{1}{2 n} I\left(\left|\xi_{0}\right|>L\left(j, \pi_{n}^{2}\right)\right) \geqslant-\left[v\left(\xi_{0}\right)+\frac{1}{2} \inf _{|y|>\left|\xi_{0}\right|}\left[w(x)-w_{*}\right]\right] \\
& +\frac{\inf _{|y|>L\left(j, \pi_{n}^{2}\right)}\left[w(x)-w_{*}\right]}{\sqrt{\inf _{|y|>L\left(j, \pi_{n}^{2}\right)}\left[w(x)-w_{*}\right]}}+n w_{*} \\
= & -u\left(\xi_{0}\right)+\frac{n}{2} \sqrt{\inf _{|y|>j}\left[w(x)-w_{*}\right]}+n w_{*} \\
\geqslant & -i n+\frac{n}{2} \sqrt{\inf _{|y|>j}\left[w(x)-w_{*}\right]}+n w_{*} .
\end{aligned}
$$

Тогда

$$
\begin{array}{r}
\frac{1}{n} \ln \mathbf{P}\left\{\int_{|x|>L\left(j, \pi_{n}^{2}\right)} \pi_{n}(d x)+\frac{1}{2 n} I\left(\left|\xi_{0}\right|>L\left(j, \pi_{n}^{2}\right)\right)>\gamma\left(L\left(j, \pi_{n}^{2}\right)\right)\right\} \\
\leqslant-i-\frac{1}{2} \sqrt{\inf _{|y|>j}\left[w(y)-w_{*}\right]}-w_{*} \rightarrow-\infty, \quad j \rightarrow \infty,
\end{array}
$$

т.е. (2.7) имеет место.

\section{Следствие. Справедливо соотношение}

$\lim _{i} \limsup _{n} \frac{1}{n} \ln \mathbf{P}\left\{\int_{(|x|>i) \cup(|y|>i)} \pi_{n}^{2}(d x, d y)>q\right\}=-\infty, \quad q>0$. 
3. Верхняя оценка в локальном п.б.у. для $\pi_{n}^{2}, n \geqslant 1$

Теорема 3.1. Пусть выполнены условия $(\mathbf{F}),\left(\mathbf{H}^{*}\right) u\left(\mathbf{H}_{0}\right)$. Тогда для любой меры $\lambda \in \mathrm{S}^{2}$

$$
\begin{aligned}
& \underset{\delta \rightarrow 0}{\limsup } \underset{n}{\lim \sup } \frac{1}{n} \ln \mathbf{P}\left\{\rho^{2}\left(\pi_{n}^{2}, \lambda\right) \leqslant \delta\right\} \\
& \leqslant-\left\{\begin{array}{lr}
\infty, & \text { мараиналь } \lambda \text { различиы, } \\
H\left(\lambda \mid \lambda_{\mu}^{\circ}\right), & \text { мараиналь } \lambda \text { совпадают }(=\mu),
\end{array}\right.
\end{aligned}
$$

где $H\left(\lambda \mid \lambda_{\mu}^{\circ}\right)-$ условная энтропия, определенная в (1.3).

Для доказательства этой теоремы используется ряд вспомогательных результатов, приводимых ниже в виде лемм.

Пусть $P$ и $Q$ - вероятностные меры на некотором измеримом про. странстве $(\Omega, \mathcal{F})$. Введем множество неотрицательных $\mathcal{F}$-измеримых функций

$$
\mathfrak{U}^{m}(Q)=\left\{u(\omega): \int_{\Omega} u(\omega) d Q=1\right\} .
$$

В соответствии с (1.3) условная энтропия $H(P \mid Q)$ меры $P$ относительно меры $Q$ определяется следуюшим образом:

$$
H(P \mid Q)= \begin{cases}\int_{\Omega} \ln \frac{d P}{d Q}(\omega) d P, & P \ll Q, \\ \infty, & \text { в остальных случаях. }\end{cases}
$$

Определим выпуклую вниз неотрицательную непрерывную функцию

$$
V(x)= \begin{cases}x \ln x+1-x, & x>0 \\ 1, & x=0\end{cases}
$$

Тогда, очевидно, имеет место формула

$$
H(P \mid Q)= \begin{cases}\int_{\Omega} V\left(\frac{d P}{d Q}(\omega)\right) d Q, & P \ll Q \\ \infty, & \text { в остальных случаях }\end{cases}
$$

Для функции $u \in \mathfrak{U}^{m}$ обозначим $G(u)=\int_{\Omega} \ln u(\omega) d P$.

Хорошо известно (см. [2]), что в этом случае

$$
H(P \mid Q)=\sup _{u \in \mathfrak{U}^{m}} G(u)
$$

Действительно, при $P \ll Q(h=d P / d Q)$ для любой функции $u \in \mathfrak{U}^{m}(Q)$ имеем $G(u)=\int_{\Omega} h \ln u d Q=\int_{\Omega}(h \ln u+1-u) d Q$ и, поскольку при $x \geqslant 0$

$$
\sup _{y \geqslant 0} x \ln y+1-y=V(x)
$$

имеет место неравенство $G(u) \leqslant \int_{\Omega} V(h) d Q=G(h)$, т.е. $\sup _{u \in \mathfrak{U}^{m}} G(u)$ достигается в точке $h \in \mathfrak{U}^{m}(Q)$. 
Если предположение $P \ll Q$ не выполняется, обозначим $P^{s}$ сингулярную часть меры $P$ относительно $Q$. Мера $P^{s}$ выделяет такое множество $\Gamma$, что $Q(\Gamma)=0$ и $P^{s}(\Omega \backslash \Gamma)=0$. Возьмем $u_{N}(\omega)=1+N I_{\Gamma}(\omega)$, принадлежащее $\mathfrak{U}^{m}(Q)$. Имеем:

$$
\begin{aligned}
G\left(u_{N}\right) & =\int_{\Omega} \ln \left(1+N I_{\Gamma}(\omega)\right) d P \geqslant \int_{\Omega} \ln \left(1+N I_{\Gamma}(\omega)\right) d P^{s} \\
& =\ln (1+N) \rightarrow \infty, \quad N \rightarrow \infty .
\end{aligned}
$$

В применении к рассматриваемому нами случағо далее будет использоваться следующая лемма.

Лемма 3.1. Пусть $\Omega=\mathbf{R}^{2}$. Тогда

$$
H(P \mid Q)=\sup _{u \in \Perp^{c}(Q)} G(u)
$$

где $\mathfrak{U}^{c}(Q)-$ подмножество $\mathfrak{U}^{m}(Q)$ непрерывньц функций.

Доказ ат ельство. Предположим, что $P \ll Q$ с $h(\omega)=$ $(d P / d Q)(\omega)$. Обозначим

$$
\begin{aligned}
f_{N}(\omega) & =h(\omega) I_{] N^{-1}, N\right]}(h(\omega))+\left(1-I_{\left.\mid N^{-1}, N\right]}(h(\omega))\right), \\
c_{N} & =\left(\int_{\Omega} f_{N} d Q\right)^{-1}, \quad h_{N}(\omega)=c_{N} f_{N}(\omega) .
\end{aligned}
$$

Очевицно, $h_{N} \in \mathfrak{U}^{m}(Q)$ и

$$
\begin{aligned}
G\left(h_{N}\right) & =\int_{\Omega} \ln h_{N}(\omega) d P=\int_{\Omega} h(\omega) \ln h_{N}(\omega) d Q \\
& =\ln c_{N}+\int_{1 / N<h(\omega) \leqslant N} h(\omega) \ln h(\omega) d Q \\
& =\ln c_{N}+\int_{1 / N<h(\omega) \leqslant 1} h(\omega) \ln h(\omega) d Q+\int_{1<h(\omega) \leqslant N} h \ln h(\omega) d Q .
\end{aligned}
$$

Поскольку $\lim _{N} c_{N}=1$, по теореме Лебега о мажорируемой сходимости

$$
\lim _{N} \int_{1 / N<h(\omega) \leqslant 1} h(\omega) \ln h(\omega) d Q=\int_{h(\omega) \leqslant 1} h(\omega) \ln h(\omega) d Q
$$

и по теореме Беппо Јеви

$$
\lim _{N} \int_{1<h(\omega) \leqslant N} h(\omega) \ln h(\omega) d Q=\int_{1<h(\omega)} h(\omega) \ln h(\omega) d Q
$$

ro

$$
\lim _{N} G\left(h_{N}\right)=G(h) .
$$

Для фиксированного $N$ выберем последовательность $u_{N, n}, n \geqslant 1$, непрерывных функций такую, что $1 /(2 N) \leqslant u_{N, n} \leqslant 2 N, Q-\lim _{n} u_{N, n}=h_{N}$ (и, 
следовательно, $\left.\lim _{n} \int_{\Omega} u_{N, n} d Q=1\right)$. Положим $\bar{u}_{N, n}=u_{N, n} / \int_{\Omega} u_{N, n} d Q$ и заметим, что $\bar{u}_{N, n} \in \mathfrak{U}^{c}(Q)$. В силу $P \ll Q, P-\lim _{n} u_{N, n}=h_{N}$. Тогда по теореме Лебега о мажорируемой сходимости $\lim _{n} G\left(u_{N, n}\right)=G\left(h_{N}\right)$. Поэтому для любого $\varepsilon>0$ найдутся величины $N(\varepsilon)$ и $n(\varepsilon)$ такие, что $G\left(h_{N(\varepsilon)}\right)+\varepsilon \geqslant G(h)$ и $G\left(\bar{u}_{N(\varepsilon), n(\varepsilon)}\right)+\varepsilon \geqslant G\left(h_{N(\varepsilon)}\right)$.

Таким образом, для любого $\varepsilon>0$ существует функция $u_{\varepsilon}=$ $\bar{u}_{N(\varepsilon), n(\varepsilon)} \in \mathfrak{U}^{c}(Q)$ со свойством $G\left(u_{\varepsilon}\right)+2 \varepsilon \geqslant G(h)=H(P \mid Q)$, т.е. при условии $P \ll Q$ требуемый результат имеет место.

Если условие $P \ll Q$ не выполняется, то при фиксированном $N$ выберем последовательность $u_{N, n}, n \geqslant 1$, с $u_{N, n} \in \mathfrak{U}^{c}, \frac{1}{2} \leqslant u_{N, n} \leqslant 2 N, n \geqslant 1$, $P^{s}-\lim _{n} u_{N, n}=u_{N}$ с определенной выше функцией $u_{N}(\omega)=1+N I_{\Gamma}(\omega)$.

Тогда

$$
\begin{aligned}
G\left(u_{N, n}\right) & =\int_{\Omega} \ln u_{N, n} d P=\int_{\Omega} \ln \left[\left(u_{N, n}+\frac{1}{2}\right)-\frac{1}{2}\right] d P \\
& \geqslant \int_{\Omega} \ln \left(u_{N, n}+\frac{1}{2}\right) d P-\ln 2 \geqslant \int_{\Omega} \ln \left(u_{N, n}+\frac{1}{2}\right) d P^{s}-\ln 2 .
\end{aligned}
$$

Следовательно, $\liminf _{n} G\left(u_{N, n}\right) \geqslant \ln \left(\frac{3}{2}+N\right)-\ln 2$, т.е. $\sup _{u \in \cup^{c}} G(u)=\infty$.

Лемма 3.2. Пусть $\lambda_{\alpha \beta}$ и $\lambda_{\mu \nu}-$ вероятностные меры из $\mathbf{S}^{2}$ с маргиналами $\alpha, \beta u \mu, \nu$. Тогда

$$
\rho^{2}\left(\lambda_{\alpha \beta}, \lambda_{\mu \nu}\right) \geqslant\left\{\begin{array}{l}
\rho(\alpha, \mu) \\
\rho(\beta, \nu)
\end{array}\right.
$$

и для любого $\delta>0$

$$
\left\{\rho^{2}\left(\lambda_{\alpha \beta}, \lambda_{\mu \nu}\right) \leqslant \delta\right\} \subseteq\{\rho(\mu, \nu) \leqslant 2 \delta+\rho(\alpha, \beta)\}
$$

Д о к а 3 а т е л ь с т в о. Обозначим $F_{\alpha \beta}$ и $F_{\mu \nu}$ функции распределения мер $\lambda_{\alpha \beta}$ и $\lambda_{\mu \nu}$. По определению $\rho^{2}\left(\lambda_{\alpha \beta}, \lambda_{\mu \nu}\right)$ и $\rho(\alpha, \mu)$ имеем:

$$
\begin{aligned}
\rho^{2}\left(\lambda_{\alpha \beta}, \lambda_{\mu \nu}\right)=\sup _{x, y} \min [u: & F_{\alpha \beta}(x-u, y-u)-u \\
& \left.\leqslant F_{\mu \nu}(x, y) \leqslant F_{\alpha \beta}(x+u, y+u)+u\right] \\
\geqslant \sup _{x} \min [u: & F_{\alpha \beta}(x-u, \infty)-u \\
& \left.\leqslant F_{\mu \nu}(x, \infty) \leqslant F_{\alpha \beta}(x+u, \infty)+u\right]=\rho(\alpha, \mu)
\end{aligned}
$$

и, аналогично, $\rho^{2}\left(\lambda_{\alpha \beta}, \lambda_{\mu \nu}\right) \geqslant \rho(\beta, \nu)$.

Следовательно,

$$
\left\{\rho^{2}\left(\lambda_{\alpha \beta}, \lambda_{\mu \nu}\right) \leqslant \delta\right\} \subseteq\{\rho(\alpha, \mu) \leqslant \delta\} \cap\{\rho(\beta, \nu) \leqslant \delta\} .
$$

По неравенству треугольника $\rho(\alpha, \beta) \leqslant \rho(\alpha, \mu)+\rho(\mu, \beta)$ и $\rho(\mu, \nu) \leqslant$ $\rho(\mu, \beta)+\rho(\nu, \beta)$ и, значит,

$$
\left\{\rho^{2}\left(\lambda_{\alpha \beta}, \lambda_{\mu \nu}\right) \leqslant \delta\right\} \subseteq\{\rho(\beta, \mu) \leqslant \delta+\rho(\alpha, \beta)\} \cap\{\rho(\beta, \nu) \leqslant \delta+\rho(\alpha, \beta)\}
$$




$$
\subseteq\{\rho(\mu, \nu) \leqslant 2(\delta+\rho(\alpha, \beta))\}
$$

Пусть $\nu_{1}$ и $\nu_{2}$ - вероятностные меры из $\mathbf{S}^{2}$ и $F_{1}$ и $F_{2}-$ их функции распределения. Обозначим $a=\rho^{2}\left(\nu_{1}, \nu_{2}\right)$ и $x=\left(x_{1}, x_{2}\right)$.

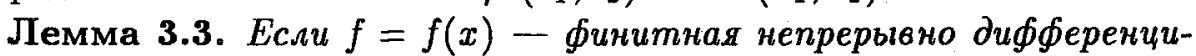
руемая функчия (по $\left.\partial^{2} /\left(\partial x_{1} \partial x_{2}\right)\right)$, то

$$
\begin{aligned}
& \left|\int_{\mathbf{R}^{2}} f(x)\left[\nu_{1}(d x)-\nu_{2}(d x)\right]\right| \\
& \quad \leqslant a \int_{\mathbf{R}^{2}}\left|\frac{\partial^{2}}{\partial x_{1} \partial x_{2}} f(x)\right| d x+\int_{\mathbf{R}^{2}}\left|\frac{\partial^{2}}{\partial x_{1} \partial x_{2}} f(x)\right|[F(x+a)-F(x-a)] d x,
\end{aligned}
$$

где $F(x)$ - любая из функций $F_{1}(x)$ или $F_{2}(x)$.

Д о к а 3 а т л в с т в о. Интегрируя по частям, получаем

$$
\int_{\mathbf{R}^{2}} f(x) d F_{i}(x)=\int_{\mathbf{R}^{2}} \frac{\partial^{2}}{\partial x_{1} \partial x_{2}} f(x) F_{i}(x) d x .
$$

Тогда

$$
\left|\int_{\mathbf{R}^{2}} f(x)\left[\nu_{1}(d x)-\nu_{2}(d x)\right]\right| \leqslant \int_{\mathbf{R}^{2}}\left|\frac{\partial^{2}}{\partial x_{1} \partial x_{2}} f(x)\right|\left[F_{1}(x)-F_{2}(x)\right] d x .
$$

По определению метрики Леви-Прохорова $F_{1}(x-a)-a \leqslant F_{2}(x)$ и $F_{2}(x) \leqslant$ $F_{1}(x+a)+a$, т.е. требуемый результат имеет место с $F=F_{1}: \mid F_{1}(x)-$ $F_{2}(x)|\leqslant a+| F_{1}(x+a)-F_{1}(x-a) \mid$.

Аналогичным образом устанавливается его справедливость при $F=F_{2}$.

Следствие. Если $f=f(x)$ - финитная непрерывная функция, по, равномерно аппрохсимируя $f(x)$ функииями $f_{\varepsilon}(x)\left(\sup _{x \in \mathbf{R}} \mid f(x)-\right.$ $\left.f_{\varepsilon}(x) \mid \leqslant \varepsilon / 2\right)$, удовлетворяюшими условию леммы, приходим к оченке

$$
\begin{gathered}
\left|\int_{\mathbf{R}^{2}} f(x)\left[\nu_{1}(d x)-\nu_{2}(d x)\right]\right| \leqslant \varepsilon+a \int_{\mathbf{R}^{2}}\left|\frac{\partial^{2}}{\partial x_{1} \partial x_{2}} f_{\varepsilon}(x)\right| d x \\
+\int_{\mathbf{R}^{2}}\left|\frac{\partial^{2}}{\partial x_{1} \partial x_{2}} f_{\varepsilon}(x)\right|[F(x+a)-F(x-a)] d x
\end{gathered}
$$

Следуюшая лемма играет важную роль при доказательстве теоремы 3.1 .

Лемма 3.4. Пусть выполнены условия $(\mathbf{F}),\left(\mathbf{H}^{*}\right)$ u $\left(\mathbf{H}_{0}\right)$. Тогда для любой мерьц $\lambda \in \mathbf{S}_{\mu \mu}$

$$
\begin{aligned}
& \lim _{q \rightarrow 0} \limsup _{i} \underset{\delta \rightarrow 0}{\limsup \limsup } \frac{1}{n} \\
& \quad \times \ln \mathbf{P}\left\{\rho^{2}\left(\pi_{n}^{2}, \lambda\right) \leqslant \delta, \int_{(|x|>i) \cup(|y|>i)} \pi_{n}^{2}(d x, d y) \leqslant q\right\} \leqslant-H\left(\lambda \mid \lambda_{\mu}^{\circ}\right) .
\end{aligned}
$$


Д о к а 3 а т е лг с с т в о. Обозначим

$$
A(n, i, q)=\left\{\rho^{2}\left(\pi_{n}^{2}, \lambda\right) \leqslant \delta, \int_{(|x|>i) \cup(|y|>i)} \pi_{n}^{2}(d x, d y) \leqslant q\right\} .
$$

Возьмем непрерывную финитную функцию $v(x, y)$ и определим

$$
u(x, y)=\frac{e^{v(x, y)}}{\int_{\mathbf{R}} e^{v(x, z)} \pi(x, d z)} .
$$

В силу условия $(\mathbf{F}), u(x, y)$ - непрерывная ограниченная функция такая, что $\int_{\mathbf{R}^{2}} u(x, y) \lambda_{\mu}^{\circ}(d x, d y)=1$, т.е. $u(x, y) \in \mathfrak{U}^{c}\left(\lambda_{\mu}^{\circ}\right)$.

Положим $Z_{n}=\prod_{k=1}^{n} u\left(\xi_{k-1}, \xi_{k}\right)$. В силу марковского свойства

$$
\mathbf{E}\left(u\left(\xi_{k-1}, \xi_{k}\right) \mid \xi_{k-1}\right)=\mathbf{E}\left(u\left(\xi_{k-1}, \xi_{k}\right) \mid \xi_{k-1}, \ldots, \xi_{0}\right) \quad \text { P-п.н., }
$$

получаем $\mathrm{E} Z_{n}=1$ и очевидное неравенство

$$
1 \geqslant \mathrm{E} I_{A(n, i, q)} Z_{n}
$$

На множестве $A(n, i, q)$ оценим снизу $\ln Z_{n}$. Имеем:

$$
\begin{aligned}
\ln Z_{n}= & \sum_{k=1}^{n} \ln u\left(\xi_{k-1}, \xi_{k}\right)=n \int_{\mathbf{R}^{2}} \ln u(x, y) \pi_{n}^{2}(d x, d y) \\
\geqslant & n \int_{\mathbf{R}^{2}} \ln u(x, y) \lambda(d x, d y) \\
& -n\left|\int_{\mathbf{R}^{2}} \ln u(x, y)\left[\lambda(d x, d y)-\pi_{n}^{2}(d x, d y)\right]\right|
\end{aligned}
$$

Выберем неотрицательную непрерывную функцию $\varphi_{i}(x, y)$ таким образом, чтобы $\varphi_{i}(x, y)=1$ на $\{|x| \leqslant i-1\} \cap\{|y| \leqslant i-1\}$ и $\varphi_{i}(x, y)=0$ на $\{|x| \geqslant i\} \cup\{|y| \geqslant i\}$. Тогда

$$
\begin{aligned}
& \ln Z_{n} \geqslant n \int_{\mathbf{R}^{2}} \ln u(x, y) \lambda(d x, d y) \\
& -n\left|\int_{\mathbf{R}^{2}} \varphi_{i}(x, y) \ln u(x, y)\left[\lambda(d x, d y)-\pi_{n}^{2}(d x, d y)\right]\right| \\
& -n \sup _{x, y}|\ln u(x, y)| \int_{(|x| \geqslant i) \cup(|y| \geqslant i)} \lambda(d x, d y) \\
& -n \int_{(|x| \geqslant i) \cup(|y| \geqslant i)} \pi_{n}^{2}(d x, d y) \\
& =n \int_{\mathbf{R}^{2}} \ln u(x, y) \lambda(d x, d y)-n r^{\prime}(i)-n r^{\prime \prime}(i)-n r^{\prime \prime \prime}(i, n) .
\end{aligned}
$$

Обозначим $F(x, y)$ функцию распределения меры $\lambda(d x, d y)$. По следствию к лемме 3.3 получаем следующие оценки на множестве $A(n, i, q)$ :

$$
r^{\prime}(i) \leqslant \varepsilon+\delta \int_{\mathbf{R}^{2}}\left|\frac{\partial^{2}}{\partial x_{1} \partial x_{2}} f_{\varepsilon}(x, y)\right| d x d y
$$




$$
\begin{gathered}
+\int_{\mathbf{R}^{2}}\left|\frac{\partial^{2}}{\partial x_{1} \partial x_{2}} f_{\varepsilon}(x, y)\right|[F(x+\delta, y+\delta)-F(x-\delta, y-\delta)] d x d y \\
\left(\leqslant \varepsilon+q^{\prime}(i, \varepsilon)\right)
\end{gathered}
$$

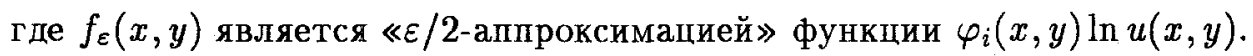
Далее,

$$
\begin{aligned}
& r^{\prime \prime}(i)=\sup _{x, y}|\ln u(x, y)| \int_{(|x| \geqslant i) \cup(|y| \geqslant i)} \lambda(d x, d y) \quad\left(=q^{\prime \prime}(i)\right), \\
& r^{\prime \prime \prime}(i, n) \leqslant \sup _{x, y}|\ln u(x, y)| q .
\end{aligned}
$$

Использование этих оценок и (3.2) приводит к неравенству

$$
\begin{aligned}
\frac{1}{n} \ln \mathbf{P}(A(n, i, q)) \leqslant & -\int_{\mathbf{R}^{2}} \ln u(x, y) \lambda(d x, d y)+\varepsilon+q^{\prime}(i, \varepsilon) \\
& +q^{\prime \prime}(i)+\sup _{x, y}|\ln u(x, y)| q,
\end{aligned}
$$

согласно которому

$\lim _{q \rightarrow 0} \limsup _{i} \limsup _{\delta \rightarrow 0} \limsup _{n} \frac{1}{n} \ln \mathbf{P}(A(n, i, q)) \leqslant \varepsilon-\int_{\mathbf{R}^{2}} \ln u(x, y) \lambda(d x, d y)$.

Таким образом, требуемый результат выводится, в силу произвольности $\varepsilon$, из леммы 3.1 , поскольку $\sup _{u \in \mathfrak{L}^{c}} \int_{\mathbf{R}^{2}} \ln u(x, y) \lambda(d x, d y)=$ $H\left(\lambda \mid \lambda_{\mu}^{\circ}\right)$.

Д ок а з а те л ь т в о т е о ем ы 3.1. Предиоложим, что мера $\lambda$ из $\mathbf{S}^{2}$ имеет разные маргиналы, скажем $\mu$ и $\nu$. Обозначим $\pi_{n}$ и $\pi_{n}^{\prime}$ маргиналы меры $\pi_{n}^{2}$ :

$$
\pi_{n}(A)=\frac{1}{n} \sum_{k=1}^{n} I\left(\xi_{k-1} \in A\right), \quad \pi_{n}^{\prime}(A)=\frac{1}{n} \sum_{k=1}^{n} I\left(\xi_{k} \in A\right) .
$$

Поскольку полная вариация $\left\|\pi_{n}-\pi_{n}^{\prime}\right\| \leqslant 2 / n$, то $\rho\left(\pi_{n}, \pi_{n}^{\prime}\right) \leqslant 2 / n$. По лемме $3.2\left\{\rho^{2}\left(\pi_{n}^{2}, \lambda\right) \leqslant \delta\right\} \subseteq\{\rho(\mu, \nu) \leqslant 2 \delta+2 / n\}$ и, значит, при $2 \delta+2 / n \leqslant$ $\rho(\mu, \nu)$ выполнено соотношение $\ln \mathrm{P}\left\{\rho\left(\pi_{n}^{2}, \lambda\right) \leqslant \delta\right\}=-\infty$, т.е. эта часть утверждения доказана.

Рассмотрим случай меры $\lambda$ из $\mathbf{S}^{2}$ с одинаковыми маргиналами. Обозначим

$$
A(n)=\left\{\rho^{2}\left(\pi_{n}^{2}, \lambda\right) \leqslant \delta\right\}
$$

и

$$
B(n, i, q)=\left\{\int_{(|x|>i) \cup(|y|>i)} \pi_{n}^{2}(d x, d y)>q\right\} .
$$

Требуемый результат вытекает из леммы 3.4, следствия к теореме 2.1 и леммы 2.2, которая может быть переформулирована следуюшим обра- 
зом: при $\lim _{q \rightarrow 0} \limsup \operatorname{simsup}_{n} \frac{1}{n} \ln \mathbf{P}(B(n, i, q))=-\infty$

$$
\begin{aligned}
& \underset{\delta \rightarrow 0}{\limsup } \limsup _{n} \frac{1}{n} \ln \mathbf{P}(A(n)) \\
& \quad=\lim _{q \rightarrow 0} \limsup _{i} \limsup _{\delta \rightarrow 0} \limsup \frac{1}{n} \ln \mathbf{P}(A(n), \Omega \backslash B(n, i, q)),
\end{aligned}
$$

если заметить, что $A(n) \cap(\Omega \backslash B(n, i, q))=A(n, i, q)$.

4. Нижняя оценка в локальном п.б.у. для $\pi_{n}^{2}, n \geqslant 1$

Теорема 4.1. Пусть өыполнены условия $\left(\mathbf{I}^{\prime}\right)$ u $\left(\mathbf{R M}^{\prime}\right)$. Тогда для любого $\lambda \in \mathbf{S}^{2}$ и $\delta>0$

$$
\liminf _{n} \frac{1}{n} \ln \mathbf{P}\left\{\rho^{2}\left(\pi_{n}^{2}, \lambda\right) \leqslant \delta\right\} \geqslant-J^{2}(\lambda) .
$$

Доказательство этой теоремы состоит из нескольких этапов, сформулированных ниже в виде лемм.

Лемма 4.1. Пусть выполнены условия $\left(\mathrm{I}^{\prime}\right) u\left(\mathbf{R M}^{\prime}\right)$. Тогда мерь $\alpha$ и $l$ әквивалентны $(\alpha \sim l)$.

Д оказа те л в с т в о. В силу условий $\left(\mathbf{I}^{\prime}\right)$ и $\left(\mathbf{R M}^{\prime}\right)$,

$$
\alpha(d y)=l(d y) \int_{\mathbf{R}} p^{\prime}(y \mid x) \alpha(d x)
$$

т.е. $\alpha \ll l$. Заметим, что $d \alpha / d l>0 l$-п.н. Тогда, в силу разложения Лебега меры $l$ относительно $\alpha$, имеет место $l \ll \alpha$.

Лемма 4.2. Пусть выполнены условия $\left(\mathbf{I}^{\prime}\right) u\left(\mathbf{R M}^{\prime}\right)$. Тогда

$$
p(x \mid y)=\frac{d \lambda^{\prime}}{d \lambda_{\alpha}^{\circ}}(x, y) p^{\prime}(y \mid x) \quad(l \times \alpha)-\text { n.r. }
$$

u $p(y \mid x)>0(l \times \alpha)-n . \mu$.

Д о к а з а те ль с т в о. В силу $\left(\mathrm{I}^{\prime}\right), \lambda^{\prime} \sim \lambda_{\alpha}^{\circ}$ и, значит,

$$
\pi(x, d y) \alpha(d x)=\frac{d \lambda_{\alpha}^{\circ}}{d \lambda^{\prime}}(x, y) p^{\prime}(y \mid x) l(d y) \alpha(d x)
$$

Первое утверждение имеет место, поскольку $\pi(x, d y)=p(y \mid x) l(d y)$ при каждом $x$. Чтобы доказать второе, достаточно показать, что $d \lambda_{\alpha}^{\circ} / d \lambda^{\prime}>0$ $(l \times \alpha)$-п.н. Воспользуемся тем, что $d \lambda_{\alpha}^{\circ} / d \lambda^{\prime}>0 \lambda^{\prime}$-п.н., $\lambda^{\prime}(d x, d y)=$ $p^{\prime}(y \mid x) l(d y) \alpha(d x)$ и $p^{\prime}(y \mid x)>0(l \times \alpha)$-п.н. Тогда $\lambda^{\prime}(A)=0$ влечет за собой $(l \times \alpha)(A)=0$, т.е. $(l \times \alpha) \ll \lambda^{\prime}$, и имеет место требуемое неравенство.

Лемма 4.3. Пусть выполнены условия $\left(\mathbf{I}^{\prime}\right) u\left(\mathbf{R M}^{\prime}\right)$ u $H\left(\lambda \mid \lambda_{\mu}^{\circ}\right)<\infty$ для $\lambda \in \mathbf{S}_{\mu \mu}$. Тогда мера $\mu$ абсолютно непрерывна относительно $l$ $(\mu \ll l)$.

Д о к а з а т е л ь с т в о. В силу $(1.3), H\left(\lambda \mid \lambda_{\mu}^{\circ}\right)<\infty$ влечет за собой $\lambda^{\prime} \ll \lambda_{\mu}^{\circ}$. По определению меры $\lambda_{\mu}^{\circ}$ и условию $\left(\mathbf{R M}^{\prime}\right)$ имеем $\lambda_{\mu}^{\circ}(d x, d y)=$ 
$p(y \mid x) l(d y) \mu(d x)$. Пусть $l(A)=0$. Тогда

$$
\lambda_{\mu}^{\circ}(\mathbf{R} \times A)=\int_{A} \int_{\mathbf{R}} p(y \mid x) \mu(d x) l(d y)=0 .
$$

Следовательно, $\mu(A)=\lambda(\mathbf{R} \times A)=0$.

Лемма 4.4. Пусть $\eta=\left(\eta_{k}\right)_{k \geqslant 0}$ есть стачионарный марковский прочесс, принимающий значения $\mathbf{6} \mathbf{R}$, с маргинальным распределением $\mu(d x)$. Если переходная вероятность $\pi_{\mu}(x, d y)$ этого марковского прочесса имеет условную плотность $p_{\mu}(y \mid x)$ относительно некоторой $\sigma$ конечной меры l:

$$
\pi_{\mu}(x, d y)=p_{\mu}(y \mid x) l(d y) \quad \mu-n . \mu ., \quad p_{\mu}(y \mid x)>0 \quad(l \times \mu)-n . \text { } .,
$$

то $\eta$ есть $\mu$-эргодический прочесс.

Д ок а з а т е ль с т в о. Согласно теореме 4 (см. $[18$, гл. $4, \S 1]$ ), $\mu$-эргодичность процесса $\eta$ имеет место, если уравнение

$$
f(x)=\int_{\mathbf{R}} f(y) \pi_{\mu}(x, d y) \quad \mu \text {-п.н. }
$$

имеет единственное $\mu$-п.н. (с точностью до мультипликативной константы) ограниченное решение.

Пусть $f(x)$ - какое-нибудь решещие уравнения (4.2). Покажем сначала, что

$$
\mu\{x: \operatorname{sign} f(x)=\text { const }\}=1 .
$$

Поскольку $\mu$ - инвариантная мера, то

$$
\mu(d y)=l(d y) \int_{\mathbf{R}} p_{\mu}(y \mid x) \mu(d x) .
$$

Из (4.2) и (4.4) вытекает, что

$$
\begin{aligned}
\int_{\mathbf{R}}|f(x)| \mu(d x) & =\int_{\mathbf{R}}\left|\int_{\mathbf{R}} f(y) p_{\mu}(y \mid x) l(d y)\right| \mu(d x) \\
& \leqslant \int_{\mathbf{R}} \int_{\mathbf{R}}|f(y)| p_{\mu}(y \mid x) l(d y) \mu(d x)=\int_{\mathbf{R}}|f(y)| \mu(d y)
\end{aligned}
$$

и, следовательно,

$$
\int_{\mathbf{R}}\left\{\int_{\mathbf{R}}|f(y)| p_{\mu}(y \mid x) l(d y)-\left|\int_{\mathbf{R}} f(y) p_{\mu}(y \mid x) l(d y)\right|\right\} \mu(d x)=0 .
$$

Последнее равенство означает, что $\mu$-п.н.

$$
\int_{f^{+}(y)>0} f^{+}(y) p_{\mu}(y \mid x) l(d y) \int_{f-(y)>0} f^{-}(y) p_{\mu}(y \mid x) l(d y)=0
$$

где $a^{+}=\max (0, a), a^{-}=-\min (a, 0)$. По лемме $4.1 l \sim \mu$. Следовательно, нарушение (4.3) противоречит (4.5), т.е. (4.3) выполняется. 
Пусть $f_{1}(x)$ и $f_{2}(x)$ - решения уравнения (4.2). Тогда $f(x)=$ $c_{1} f_{1}(x)+c_{2} f_{2}(x)$ также является решением этого уравнения при любых константах $c_{1}$ и $c_{2}$, которые могут быть выбраны таким образом, чтобы нарушить (4.3). Значит (4.2) имеет единственное решение.

Лемма 4.5. Пусть выполнены условия $\left(\mathrm{I}^{\prime}\right)$ u $\left(\mathbf{R M}^{\prime}\right)$. Для любой мерь $\lambda \in \mathbf{S}_{\mu \mu}$ с $H\left(\lambda \mid \lambda_{\mu}^{\circ}\right)<\infty$ существуют семейства $\lambda^{\varepsilon} \in \mathbf{S}^{2}, \mu(\varepsilon) \in$ $\mathbf{S}, \varepsilon \in(0,1)$, такие, что $\lambda^{\varepsilon} \in \mathbf{S}_{\mu(\varepsilon) \mu(\varepsilon)}$ при любом $\varepsilon u$

1. $\lambda^{\varepsilon} \sim \lambda_{\mu(\varepsilon)}^{\circ}$,

2. $H\left(\lambda^{\varepsilon} \mid \lambda_{\mu(\varepsilon)}^{\circ}\right)<\infty$,

3. $\lim _{\varepsilon \rightarrow 0} \rho^{2}\left(\lambda^{\varepsilon}, \lambda\right)=0$,

4. $\lim _{\varepsilon \rightarrow 0} H\left(\lambda^{\varepsilon} \mid \lambda_{\mu(\varepsilon)}^{\circ}\right)=H\left(\lambda \mid \lambda_{\mu}^{\circ}\right)$.

Д о к а 3 а т е л ь с т в о. Пусть $\lambda^{\prime}-$ мера из условия $\left(I^{\prime}\right)$ с маргиналами $\alpha$. Положим

$$
\mu(\varepsilon)=(1-\varepsilon) \mu+\varepsilon \alpha, \quad \lambda^{\varepsilon}=(1-\varepsilon) \lambda+\varepsilon \lambda^{\prime}, \quad \lambda_{\mu(\varepsilon)}^{\circ}=(1-\varepsilon) \lambda_{\mu}^{\circ}+\varepsilon \lambda_{\alpha}^{\circ}
$$

и заметим, что маргиналами мер $\lambda^{\varepsilon}$ являются $(1-\varepsilon) \mu+\varepsilon \alpha=\mu(\varepsilon)$.

1. Пусть $\lambda_{\mu(\varepsilon)}^{\circ}(A)=0$. Тогда $\lambda_{\mu}^{\circ}(A)=0$ и $\lambda_{\alpha}^{\circ}(A)=0$. Поскольку $H\left(\lambda \mid \lambda_{\mu}^{\circ}\right)<\infty$, то $\lambda \ll \lambda_{\mu}^{\circ}$ и, значит, $\lambda(A)=0$. По условию $\left(\mathbf{I}^{\prime}\right) \lambda^{\prime} \sim \lambda_{\alpha}^{\circ}$, что влечет за собой $\lambda^{\prime}(A)=0$. Следовательно, $\lambda^{\varepsilon}(A)=0$.

$\mathrm{C}$ другой стороны, если $\lambda^{\varepsilon}(A)=0$, то $\lambda(A)=0$ и $\lambda^{\prime}(A)=0$, a из эквивалентности мер $\lambda^{\prime}$ и $\lambda_{\alpha}^{\circ}$ следует, что $\lambda_{\alpha}^{\circ}(A)=0$. Поэтому равенство $\lambda_{\mu(\varepsilon)}^{\circ}(A)=0$ будет иметь место, если $\lambda_{\mu}^{\circ}(A)=0$. Последнее равенство выполняется, поскольку $\lambda_{\alpha}^{\circ}(A)=0$ и, в силу представлений $\lambda_{\alpha}^{\circ}(d x, d y)=p(y \mid x) l(d y) \alpha(d x)$ и $\lambda_{\mu}^{\circ}(d x, d y)=p(y \mid x) l(d y) \mu(d x)$ и абсолютной непрерывности $\mu \ll \alpha$ (лемма 4.3)), имеет место $\lambda_{\mu}^{\circ} \ll \lambda_{\alpha}^{\circ}$.

Итак, $\lambda^{\varepsilon} \sim \lambda_{\mu(\varepsilon)}^{\circ}$.

2. Очевидно, $\mu \ll \mu(\varepsilon)$ и $\alpha \ll \mu(\varepsilon)$. Обозначим

$$
\begin{aligned}
h_{\alpha}(x, y) & =\frac{d \lambda^{\prime}}{d \lambda_{\alpha}^{\circ}}(x, y), \quad h(x, y)=\frac{d \lambda}{d \lambda_{\mu}^{\circ}}(x, y), \\
g_{\mu}(x) & =(1-\varepsilon) \frac{d \mu}{d \mu(\varepsilon)}(x), \quad g(x)=\varepsilon \frac{d \alpha}{d \mu(\varepsilon)}(x) .
\end{aligned}
$$

Тогда

$$
\frac{d \lambda(\varepsilon)}{d \lambda_{\mu(\varepsilon)}^{\circ}}(x, y)=h(x, y) g(x)+h_{\alpha}(x, y) g_{\mu}(x) \quad\left(=h^{\varepsilon}(x, y)\right) .
$$

По определению условной энтропии (см. (3.1))

$$
H\left(\lambda^{\varepsilon} \mid \lambda_{\mu(\varepsilon)}^{\circ}\right)=\int_{\mathbf{R}^{2}} V\left(h^{\varepsilon}(x, y)\right) \lambda_{\mu(\varepsilon)}^{\circ}(d x, d y) .
$$

Из определения функций $g(x)$ и $g_{\alpha}(x)$ следует, что $g(x)+g_{\alpha}(x)=1$ $\mu(\varepsilon)$-п.н. и, значит, $\lambda_{\mu(\varepsilon)}^{\text {o }}$-п.н. Функция $V(x)$ выпукла вниз. Поэтому по 
неравенству Йенсена

$$
V\left(h^{\varepsilon}(x, y)\right) \leqslant g_{\mu}(x) V(h(x, y))+g(x) V\left(h_{\alpha}(x, y)\right) \quad \lambda_{\mu(\varepsilon)}^{\circ} \text {-п.н. }
$$

и, следовательно, с учетом (3.1) имеет место неравенство

$$
\begin{aligned}
H\left(\lambda^{\varepsilon} \mid \lambda_{\mu(\varepsilon)}^{\circ}\right) \leqslant & (1-\varepsilon) \int_{\mathbf{R}^{2}} V(h(x, y)) \frac{d \mu}{d \mu(\varepsilon)} \lambda_{\mu(\varepsilon)}^{\circ}(d x, d y) \\
& +\varepsilon \int_{\mathbf{R}^{2}} V\left(h_{\alpha}(x, y)\right) \frac{d \alpha}{d \mu(\varepsilon)} \lambda_{\mu(\varepsilon)}^{\circ}(d x, d y) \\
= & (1-\varepsilon) \int_{\mathbf{R}^{2}} V(h(x, y)) \lambda_{\mu}^{\circ}(d x, d y) \\
& +\varepsilon \int_{\mathbf{R}^{2}} V\left(h_{\alpha}(x, y)\right) \lambda_{\alpha}^{\circ}(d x, d y) \\
= & (1-\varepsilon) H\left(\lambda \mid \lambda_{\mu}^{\circ}\right)+\varepsilon H\left(\lambda^{\prime} \mid \lambda_{\alpha}^{\circ}\right)<\infty .
\end{aligned}
$$

3. Из определения $\lambda^{\varepsilon}$ получаем, что полная вариация $\left\|\lambda^{\varepsilon}-\lambda\right\| \leqslant 2 \varepsilon$, т.e. $\rho^{2}\left(\lambda^{\varepsilon}, \lambda\right) \rightarrow 0, \varepsilon \rightarrow 0$.

4. Неравенство (4.8) влечет за собой неравенство

$$
\limsup _{\varepsilon \rightarrow 0} H\left(\lambda^{\varepsilon} \mid \lambda_{\mu(\varepsilon)}^{\circ}\right) \leqslant H\left(\lambda \mid \lambda_{\mu}^{\circ}\right) .
$$

Обратное неравенство $\liminf \sin _{\varepsilon \rightarrow 0} H\left(\lambda^{\varepsilon} \mid \lambda_{\mu(\varepsilon)}^{\circ}\right) \geqslant H\left(\lambda \mid \lambda_{\mu}^{\circ}\right)$ выводится из (4.6) с помощью леммы Фату.

Д ок аз а те ль с т в о т е о ре мы 4.1. Очевидно, что в проверке нуждается лишь случай $J^{2}(\lambda)<\infty$. В данной ситуации $J^{2}(\lambda)=$ $H\left(\lambda \mid \lambda_{\mu}^{\circ}\right)$ с $\lambda \in \mathbf{S}_{\mu \mu}$ и $\lambda \ll \lambda_{\mu}^{\circ}$.

Предположим сначала, что

$$
\lambda \sim \lambda_{\mu}^{\circ}, \quad \alpha_{0} \sim \alpha
$$

и обозначим

$$
h(x, y)=\frac{d \lambda}{d \lambda_{\mu}^{\circ}}(x, y) .
$$

На измеримом пространстве $\left(\mathbf{R}^{\infty}, \mathcal{B}\left(\mathbf{R}^{\infty}\right)\right)\left(\mathbf{R}^{\infty}=\left(x_{0}, x_{1}, \ldots\right), \mathcal{B}\left(\mathbf{R}^{\infty}\right)-\right.$ борелевская $\sigma$-алгебра на $\mathbf{R}^{\infty}$ ) зададим вероятностную меру $\mathbf{Q}$, являюшуюся распределением исходного марковского процесса $\xi$, и вероятностную меру $\mathbf{Q}^{\mu}$, отвечающую стационарному марковскому процессу с инвариантной мерой $\mu$ и переходной вероятностью

$$
\pi_{\mu}(x, d y)=h(x, y) \pi(x, d y) \quad(=h(x, y) p(y \mid x) l(d y)) .
$$

В силу (4.9) и леммы $4.3 \mu \ll \alpha_{0}$ и $p_{\mu}(y \mid x)>0(l \times \mu)$-п.н. Поэтому по лемме 4.4 процесс $\left(x_{k}, \mathbf{Q}^{\mu}\right)_{k \geqslant 0}$ является $\mu$-эргодическим. Обозначим $\mathbf{Q}_{n}$ и $\mathbf{Q}_{n}^{\mu}$ сужения мер $\mathbf{Q}$ и $\mathbf{Q}^{\mu}$ на $\sigma$-алгебру $\mathcal{B}\left(\mathbf{R}^{n+1}\right)$. Поскольку $\mu \ll \alpha_{0}$, то

$$
\mathbf{Q}_{n}^{\mu} \ll \mathbf{Q}_{n}, \quad n \geqslant 0,
$$


и процесс локальной плотности $\left(3_{n}\left(x_{0}^{n}\right)\right)_{n \geqslant 0}$ меры $\mathbf{Q}^{\mu}$ относительно $\mathbf{Q}$ задается формулой

$$
\mathfrak{z}_{n}\left(x_{0}^{n}\right)=\frac{d \mu}{d \alpha_{0}}\left(x_{0}\right) \exp \left(\sum_{k=1}^{n} \ln h\left(x_{k-1}, x_{k}\right)\right) .
$$

Случайные величины $z_{n}\left(x_{0}^{n}\right)$ положительны $\mathbf{Q}^{\mu}$-п.н. и в соответствии с разложением Лебега меры $\mathbf{Q}_{n}$ относительно $\mathbf{Q}_{n}^{\mu}$ имеет место неравенство: для любого $\Delta \in \mathcal{B}\left(\mathbf{R}^{n+1}\right)$

$$
\mathbf{Q}(\Delta) \geqslant \int_{\Delta} \mathfrak{z}^{-1}\left(x_{0}^{n}\right) d \mathbf{Q}_{n}^{\mu}
$$

Введем оккупационные меры $\pi_{n}^{2}\left(x_{0}^{n}\right)$ с $\pi_{n}^{2}(A \times B)\left(x_{0}^{n}\right)=\frac{1}{n} \sum_{k=1}^{n} I\left(x_{k-1} \in\right.$ $\left.A, x_{k} \in B\right)$, определим множества

$$
\begin{aligned}
\Delta_{n}^{1} & =\left\{\rho^{2}\left(\pi_{n}^{2}\left(x_{0}^{n}\right), \lambda\right) \leqslant \delta\right\}, \\
\Delta_{n}^{2} & =\left\{\left|\frac{1}{n} \sum_{k=1}^{n} h\left(x_{k-1}, x_{k}\right)-H\left(\lambda \mid \lambda_{\mu}^{\circ}\right)\right| \leqslant \beta\right\}, \\
\Delta^{c} & =\left\{\frac{d \mu}{d \alpha_{0}}\left(x_{0}\right)>\frac{1}{c}\right\},
\end{aligned}
$$

и положим $\Delta^{n, c}=\Delta_{n}^{1} \cap \Delta_{n}^{2} \cap \Delta^{c}$. Тогда, с учетом (4.13), получаем, что

$$
\begin{aligned}
\mathbf{P}\left\{\rho_{n}^{2}\left(\pi_{n}^{2}, \lambda\right) \leqslant \delta\right\} & =\mathbf{Q}\left\{\rho_{n}^{2}\left(\pi_{n}^{2}\left(x_{0}^{n}\right), \lambda\right) \leqslant \delta\right\} \geqslant \int_{\Delta^{n, c}} z_{n}^{-1}\left(x_{0}^{n}\right) d \mathbf{Q}_{n}^{\mu} \\
& \geqslant c \mathbf{Q}_{n}^{\mu}\left(\Delta^{n, c}\right) \exp \left\{-n H\left(\lambda \mid \lambda_{\mu}^{\circ}\right)-n \beta\right\} .
\end{aligned}
$$

Отскода следует, что

$$
\liminf _{n} \ln \mathbf{P}\left\{\rho^{2}\left(\pi_{n}^{2}, \lambda\right) \leqslant \delta\right\} \geqslant-H\left(\lambda \mid \lambda_{\mu}^{\circ}\right)-\beta+\liminf _{n} \ln \mathbf{Q}_{n}^{\mu}\left(\Delta^{n, c}\right) .
$$

В силу произвольности $\beta$ требуемая нижняя оценка будет иметь место, если

$$
\lim _{c \rightarrow \infty} \mu\left\{x_{0}: \frac{d \mu}{d \alpha}\left(x_{0}\right)>\frac{1}{c}\right\}=1, \quad \lim _{n}\left\{\begin{array}{l}
\mathbf{Q}_{n}^{\mu}\left(\Delta_{n}^{1}\right) \\
\mathbf{Q}_{n}^{\mu}\left(\Delta_{n}^{2}\right)
\end{array}=1 .\right.
$$

Первое соотношение в (4.15) имеет место, поскольку, как уже отмечалось выше, $\mu \ll \alpha_{0}$. Второе соотношение в (4.15) выполнено в силу $\mu$-эргодичности процесса $\left(x_{k}, \mathbf{Q}^{\mu}\right)_{k \geqslant 0}$. Действительно, этот процесс имеет двумерное распределение $\lambda$ и, значит, в соответствии с теоремой Биркгофа-Хинчина $\lim _{n} \rho^{2}\left(\pi_{n}^{2}\left(x_{0}^{n}\right), \lambda\right)=0 \mathbf{Q}^{\mu}$-п.н., т.е. первая часть второго соотношения выполнена. По предположению

$$
\infty>H\left(\lambda \mid \lambda_{\mu}^{\circ}\right)=\int_{\mathbf{R}^{2}} \ln h(x, y) \lambda(d x, d y)
$$


и, значит, по той же теореме Биркгофа-Хинчина выполняется и вторая часть второго соотношения в (4.15).

Итак, при условии (4.9) требуемая нижняя оценка имеет место.

Пусть в (4.9) выполнено лишь второе условие: $\alpha_{0} \sim \alpha$. Возьмем меры $\lambda^{\varepsilon}$ и $\mu(\varepsilon)$ из леммы 4.5. Для мер $\lambda^{\varepsilon}$ и $\lambda_{\mu(\varepsilon)}^{\varepsilon}$ выполнено первое условие в (4.9). Покажем теперь, что $\mu(\varepsilon) \ll \alpha_{0}$. В силу лемм 4.1 и 4.3 , $\mu \ll \alpha$. Тотда (см. определение $\mu(\varepsilon)$ в лемме 4.5$) \mu(\varepsilon) \ll \alpha$, что влечет за собой требуемую абсолютную непрерывность в силу второго условия в (4.9).

\section{По доказанному}

$$
\liminf _{n} \frac{1}{n} \ln \mathbf{P}\left\{\rho^{2}\left(\pi_{n}^{2}, \lambda^{\varepsilon}\right) \leqslant \delta\right\} \geqslant-H\left(\lambda^{\varepsilon} \mid \lambda_{\mu(\varepsilon)}^{\circ}\right) .
$$

По лемме 4.5 можно выбрать $\varepsilon_{0}$ такое, что при фиксированном $\delta$ и $\varepsilon \leqslant \varepsilon_{0}$ выполняется неравенство $\rho^{2}\left(\lambda, \lambda^{\varepsilon}\right) \leqslant \delta / 2$. Тогда, с учетом леммы 4.5 ,

$$
\begin{aligned}
\liminf _{n} \frac{1}{n} \ln \mathbf{P}\left\{\rho^{2}\left(\pi_{n}^{2}, \lambda\right) \leqslant \delta\right\} & \geqslant \liminf _{n} \frac{1}{n} \ln \mathbf{P}\left\{\rho^{2}\left(\pi_{n}^{2}, \lambda^{\varepsilon}\right) \leqslant \delta / 2\right\} \\
& \geqslant-H\left(\lambda^{\varepsilon} \mid \lambda_{\mu(\varepsilon)}^{\circ}\right) \longrightarrow-H\left(\lambda \mid \lambda_{\mu}^{\circ}\right), \quad \varepsilon \rightarrow 0,
\end{aligned}
$$

т.е. требуемое неравенство имеет место при выполнении лишь второго условия в (4.9).

Чтобы отказаться и от этого условия, введем, следуя Донскеру и Варадану [1], новый марковский процесс $\xi^{1}=\left(\xi_{k}^{1}\right)_{k \geqslant 0}$ с $\xi_{k}^{1} \equiv \xi_{k+1}$. У этого марковского прощесса та же переходная вероятность $\pi(x ; d y)$ и начальное распределение

$$
\alpha_{0}^{1}(d y)=\int_{\mathbf{R}} \pi(x, d y) \alpha_{0}(d x)=l(d y) \int_{\mathbf{R}} p(y \mid x) \alpha_{0}(d x)
$$

Отсюда и в силу условия $\left(\mathrm{H}_{0}\right)$ вытекает, что $\alpha_{0}^{1} \sim l$ и, значит, в силу леммы $4.1, \alpha_{0} \sim \alpha$. Поэтому по предшествуюшему доказательству нижняя оценка имеет место для $\pi_{n}^{2}\left(\xi^{1}\right)$, где

$$
\pi_{n}^{2}\left(A \times B\left(\xi^{1}\right)=\frac{1}{n} \sum_{k=1}^{n} I\left(\xi_{k-1}^{1} \in A, \xi_{k}^{1} \in B\right)=\frac{1}{n} \sum_{k=1}^{n} I\left(\xi_{k} \in A, \xi_{k+1} \in B\right)\right.
$$

T.e.

$$
\liminf _{n} \frac{1}{n} \ln \mathbf{P}\left\{\rho^{2}\left(\pi_{n}^{2}\left(\xi^{1}\right), \lambda\right) \leqslant \delta\right\} \geqslant-H\left(\lambda \mid \lambda_{\mu}^{\circ}\right)
$$

Требуемый результат вытекает теперь из (4.16) и того факта, что полная вариация $\left\|\pi_{n}^{2}-\pi_{n}^{2}\left(\xi^{1}\right)\right\| \leqslant 2 / n$.

\section{5. Доказательство теоремы 1.2}

По теореме 2.1 семейство $\left(\pi_{n}^{2}\right)$ экспоненциально плотно. Тогда по теореме Пухальского (см. (1.9)) существует подпоследовательность $\left(\pi_{\tilde{n}}^{2}\right)$, 
удовлетворяюшая п.б.у. с функционалом действия $\tilde{J}^{2}(\lambda)$ и, значит, для $\delta>0$ имеют место верхние и нижние оценки:

$$
\begin{aligned}
& \liminf _{\widetilde{n}} \frac{1}{\widetilde{n}} \ln \mathbf{P}\left\{\rho^{2}\left(\pi_{\tilde{n}}^{2}, \lambda\right) \leqslant \delta\right\} \geqslant \liminf _{\widetilde{n}} \frac{1}{\widetilde{n}} \ln \mathbf{P}\left\{\rho^{2}\left(\pi_{\tilde{n}}^{2}, \lambda\right)<\delta\right\} \\
& \geqslant-\inf _{\nu: \rho^{2}(\nu, \lambda)<\delta} \tilde{J}^{2}(\nu) \geqslant-\tilde{J}^{2}(\nu), \\
& \underset{\tilde{n}}{\limsup } \frac{1}{\widetilde{n}} \ln \mathbf{P}\left\{\rho^{2}\left(\pi_{\tilde{n}}^{2}, \lambda\right) \leqslant \delta\right\} \leqslant-\inf _{\nu: \rho^{2}(\nu, \lambda)<\delta} \tilde{J}^{2}(\nu) .
\end{aligned}
$$

По определению inf для любых $\varepsilon>0, \delta>0$ найдется мера $\nu_{\varepsilon}^{\delta}$ такая, что $\rho^{2}\left(\nu_{\varepsilon}^{\delta}, \lambda\right) \leqslant \delta$ и

$$
\inf _{\nu: \rho^{2}(\nu, \lambda)<\delta} \tilde{J}^{2}(\nu) \geqslant \tilde{J}^{2}\left(\nu_{\varepsilon}^{\delta}\right)-\varepsilon
$$

В силу полунепрерывности снизу функщии $\tilde{J}^{2}(\lambda)$ получаем

$$
\liminf _{\delta \rightarrow 0} \tilde{J}^{2}\left(\nu_{\varepsilon}^{\delta}\right) \geqslant \tilde{J}^{2}(\lambda)
$$

и, значит, согласно (5.2) и (5.3),

$$
\begin{aligned}
\limsup _{\tilde{n}} \frac{1}{\widetilde{n}} \ln \mathbf{P}\left\{\rho^{2}\left(\pi_{\tilde{n}}^{2}, \lambda\right) \leqslant \delta\right\} & \leqslant \limsup _{\delta \rightarrow 0}\left[\varepsilon-\tilde{J}^{2}\left(\nu_{\varepsilon}^{\delta}\right)\right]=\varepsilon-\liminf _{\delta \rightarrow 0} \tilde{J}^{2}\left(\nu_{\varepsilon}^{\delta}\right) \\
& \leqslant \varepsilon-\tilde{J}^{2}(\lambda) \rightarrow-\tilde{J}^{2}(\lambda), \quad \varepsilon \rightarrow 0 .
\end{aligned}
$$
$\left(\pi_{\tilde{n}}^{2}\right)$.

Таким образом, из (5.1) и (5.4) получаем локальный п.б.у. (1.10) для

Как отмечалось во введении (см. (1.12)), имеет место тождество $\tilde{J}^{2}(\lambda) \equiv J^{2}(\lambda)$. Используя этот факт, установим верхнюю и нижнюю оценки больших уклонений для $\left(\pi_{n}^{2}\right)$.

Для замкнутого множества $F^{2} \in \mathbf{S}^{2}$ выберем такую подпоследовательность $\left(\pi_{n^{\prime}}^{2}\right)$ подпоследовательности $\left(\pi_{n}^{2}\right)$, что

$$
\underset{n}{\limsup } \frac{1}{n} \ln \mathbf{P}\left\{\pi_{n}^{2} \in F^{2}\right\}=\lim _{n^{\prime}} \frac{1}{n^{\prime}} \ln \mathbf{P}\left\{\pi_{n^{\prime}}^{2} \in F^{2}\right\} .
$$

По теореме Пухальского (см. (1.9)) можно выбрать подпоследовательность $\left(\pi_{\tilde{n}}^{2}\right)$ подпоследовательности $\left(\pi_{n^{\prime}}^{2}\right)$ такую, что $\left(\pi_{\widetilde{n}}^{2}\right)$ удовлетворяет п.б.у. с функционалом действия $\tilde{J}^{2}(\lambda) \equiv J^{2}(\lambda)$. Тогда

$$
\begin{aligned}
\limsup _{n} \frac{1}{n} \ln \mathbf{P}\left\{\pi_{n}^{2} \in F^{2}\right\} & =\lim _{n^{\prime}} \frac{1}{n^{\prime}} \ln \mathbf{P}\left\{\pi_{n^{\prime}}^{2} \in F^{2}\right\} \\
& =\lim _{\widetilde{n}} \frac{1}{\widetilde{n}} \ln \mathbf{P}\left\{\pi_{\widetilde{n}}^{2} \in F^{2}\right\} \leqslant-\inf _{\lambda \in F^{2}} J^{2}(\lambda) .
\end{aligned}
$$

Нижняя оценка устанавливается аналогичным образом. 


\section{6. Доказательство теоремы 1.1}

Пусть $\lambda^{1}, \lambda^{2} \in \mathbf{S}^{2}$. Маргиналы этих мер обозначим $\left(\mu^{1}, \nu^{1}\right),\left(\mu^{2}, \nu^{2}\right)$. По лемме 3.2

$$
\rho^{2}\left(\lambda^{1}, \lambda^{2}\right) \geqslant\left\{\begin{array}{l}
\rho\left(\mu^{1}, \mu^{2}\right) \\
\rho\left(\nu^{1}, \nu^{2}\right)
\end{array} .\right.
$$

Поэтому отображение $\lambda^{1} \longrightarrow\left(\mu^{1}, \nu^{1}\right)$ является равномерно непрерывным в метрике Леви-Прохорова. Отсюда, согласно методу непрерывного отображения Варадана [3], следует, что семейство $\left(\pi_{n}, n \geqslant 1\right)$ удовлетворяет п.б.у. в $(\mathbf{S}, \rho)$ с функционалом действия $J^{\prime}(\mu)=\inf J^{2}(\lambda)$, где inf берется по всем мерам $\lambda \in \mathbf{S}^{2}$ хотя бы с одним маргиналом $\mu$. Поскольку $J^{2}(\lambda)=\infty$ для мер $\lambda$ с различными маргиналами, то

$$
J^{\prime}(\mu)=\inf _{\lambda \in \mathbf{S}_{\mu \mu}} H\left(\lambda \mid \lambda_{\mu}^{\circ}\right)
$$

\section{7. Пример}

Рассмотрим марковский процесс $\xi=\left(\xi_{k}\right)_{k \geqslant 0}$, заданный рекуррентным соотношением

$$
\xi_{k+1}=f\left(\xi_{k}\right)+g\left(\xi_{k}\right) \zeta_{k+1}
$$

где $\left(\zeta_{k}\right)_{k \geqslant 1}$ - последовательность независимых и одинаково распределенных случайных величин, $\xi_{0}-$ независимая от $\left(\zeta_{k}\right)_{k \geqslant 1}$ случайная величина.

Предположим, что выполнены следующие условия:

(1) $f(x), g(x)$ - непрерывные функции,

$$
\left|\frac{f(x)}{x}\right| \leqslant a<1, \quad \frac{1}{r} \leqslant|g(x)| \leqslant r, \quad r>0 ;
$$

(2) случайная величина $\zeta_{1}$ имеет плотность $p_{\zeta}(y)$ (относительно меры Лебега), $p_{\zeta}(y)$ - непрерывная функция с

$$
\begin{aligned}
& r_{1} \exp \left(-r_{2}|y|^{q}\right) \leqslant p_{\zeta}(y) \leqslant r_{3} \exp \left(-r_{4}|y|\right), \\
& r_{i}>0, \quad i=1, \ldots, 4, \quad q \geqslant 1 ;
\end{aligned}
$$

(3) $\exists$ b $>0: \mathbf{E} e^{b\left|\xi_{0}\right|}<\infty$.

Покажем, что при выполнении этих условий для процесса $\xi$ выполняется п.б.у. в $\left(\mathbf{S}^{2}, \rho^{2}\right)$ и $(\mathbf{S}, \rho)$.

В соответствии с теоремами 1.1 и 1.2 достаточно установить импликацию

$$
\{(1),(2),(3)\} \Longrightarrow\left\{(\mathbf{F}),\left(\mathbf{I}^{\prime}\right),\left(\mathbf{H}^{*}\right),\left(\mathbf{R M}^{\prime}\right),\left(\mathbf{H}_{0}\right)\right\} .
$$

В силу (2) переходная вероятность $\pi(x, d y)$ процесса $\xi$ при каждом $x$ имеет плотность по мере Лебега

$$
p(y \mid x)=p_{\zeta}\left(\frac{y-f(x)}{g(x)}\right)
$$


Поэтому $\{(\mathbf{1}),(\mathbf{2})\} \Longrightarrow\{(\mathbf{F})\}$.

В качестве меры $\lambda^{\prime}$ возьмем двумерное распределение стационарного марковского гауссовского процесса $\left(\xi_{k}^{\prime}\right)_{k \geqslant 0}$ с $\xi_{k+1}^{\prime}=a \xi_{k}^{\prime}+\zeta_{k+1}^{\prime}$, где $\left(\zeta_{k}^{\prime}\right)_{k \geqslant 1}$ - последовательность независимых и одинаково распределенных гауссовских случайных величин с параметрами $(0,1), \xi_{0}^{\prime}-$ независимая от $\left(\zeta_{k}^{\prime}\right)_{k \geqslant 1}$ гауссовская случайная величина с параметрами $\left(0,1 /\left(1-a^{2}\right)\right)$. Тогда

$$
\frac{d \alpha}{d x}(x)=\sqrt{\frac{1-a^{2}}{2 \pi}} \exp \left\{-\frac{x^{2}}{2 /\left(1-a^{2}\right)}\right\}
$$

и

$$
\lambda^{\prime}(d x, d y)=\frac{1}{\sqrt{2 \pi}} \exp \left\{-\frac{(y-a x)^{2}}{2}\right\} d y \alpha(d x) .
$$

С другой стороны, в силу $(7.3), \lambda^{\prime}(d x, d y)=p_{\zeta}((y-f(x)) / g(x)) d y \alpha(d x)$ и, значит, $\lambda^{\prime} \sim \lambda_{\alpha}^{\circ}$ с

$$
\frac{d \lambda^{\prime}}{d \lambda_{\alpha}^{\circ}}(x, y)=\frac{\sqrt{\left(1-a^{2}\right) /(2 \pi)} \exp \left\{-(y-a x)^{2} / 2\right\}}{p_{\zeta}((y-f(x)) / g(x))} .
$$

Тогда, в соответствии с (1) и (2)

$$
\frac{d \lambda^{\prime}}{d \lambda_{\alpha}^{\circ}}(x, y) \leqslant \frac{1}{r_{1}} \exp \left\{r_{2}\left|\frac{y-f(x)}{g(x)}\right|^{q}\right\} \leqslant \frac{1}{r_{1}} \exp \left\{\frac{r_{2}}{r}[|y|+a|x|]^{q}\right\}
$$

что влечет за собой

$$
H\left(\lambda^{\prime} \mid \lambda_{\alpha}^{\circ}\right) \leqslant-\ln r_{1}+\int_{\mathbf{R}^{2}} \frac{r_{2}}{r}[|y|+a|x|]^{q} \lambda^{\prime}(d x, d y)<\infty,
$$

T.e. $\left.\{(1),(2)\} \Longrightarrow\left\{\left(I^{\prime}\right)\right)\right\}$.

Для проверки $\left(\mathbf{H}^{*}\right)$ возьмем $v(x)=c|x| c c \in\left(a r_{4} / r, r_{4} / r\right)$. Тогда

$$
w(x)=c|x|-\ln \int_{\mathbf{R}} \exp (c|y|) p_{\zeta}\left(\frac{y-f(x)}{g(x)}\right) d y .
$$

Отсюда и в силу (2) приходим к неравенству

$$
\begin{aligned}
w(x) & \geqslant-\ln r_{3}+c|x|-\ln \int_{\mathbf{R}} \exp \left\{c|y|-r_{4}\left|\frac{y-f(x)}{g(x)}\right|\right\} d y \\
& \geqslant-\ln r_{3}+c|x|-\ln \int_{\mathbf{R}} \exp \left\{c|y|-\frac{r_{4}}{r}|y|+a \frac{r_{4}}{r}|x|\right\} d y \\
& =\left(c-a \frac{r_{4}}{r}\right)|x|-\ln r_{3}-\ln \int_{\mathbf{R}} \exp \left\{-\left(\frac{r_{4}}{r}-c\right)|y|\right\} d y,
\end{aligned}
$$

т.е. $\{(\mathbf{2})\} \Longrightarrow\left\{\left(\mathbf{H}^{*}\right)\right\}$. Кроме того, $\{(\mathbf{2})\} \Longrightarrow\left\{\left(\mathbf{R M}^{\prime}\right)\right\}$ и $\{(\mathbf{2}),(\mathbf{3})\} \Longrightarrow$ $\left\{\left(\mathbf{H}_{0}\right)\right\}$. 


\section{СПИСОК ЛИТЕРАТУРЫ}

1. Donsker M. D., Varadhan S. R. S. Asymptotic evaluation of certain Markov process expectations for large time. III. - Commun. Pure Appl. Math., 1976, v. 29, № 4, p. 389-461.

2. Donsker M. D., Varadhan $S . R$. S. Asymptotic evaluation of certain Markov process expectations for large time. I. - Commun. Pure Appl. Math., 1975, v. 28, № 1, p. 1-47.

3. Varadhan S. R. S. Large Deviations and Applications. Philadelphia: SIAM, 1984.

4. Donsker M. D., Varadhan S. R. S. Asymptotic evaluation of certain Markov process expectations for large time. IV. - Commun. Pure Appl. Math., 1983, v. 36, № 2, p. 183-212.

5. Ellis $R$. S. Large deviations for the empirical of a Markov chain with an application to the multivariate empirical measure. - Ann. Probab., 1988, v. 16, № 4, p. 1496-1508.

6. Dupuis P., Ellis R. S. A stochastic optimal control approach to the theory of large deviations. - Preprint, 1992.

7. Deuschel J. D., Stroock D. W. Large Deviations. New York: Academic Press, 1989.

8. Puhalskii A. A. On functional principle of large deviations. - In: New Trends in Probability and Statistics. /Ed. by V. Sazonov and T. Shervashidze. Utrecht/Vilnius: VSP/Mokslas, 1991, p. 198-218.

9. Вентиель А. Д., Фрейдлин $М$. И. Флуктуации в динамических системах под действием малых случайных возмущений. М.: Наука, 1979, 424 с.

10. Liptser R. S., Pukhalskii A. A. Limit theorems on large deviations for semimartingales. - Stochastics and Stoch. Reports, 1992, v. 38, p. 201-249.

11. De Acosta A. Large deviations for empirical measures of Markov chains. - J. Theoret. Probab., 1990, v. 3, p. 395-431.

12. Гертнер Ю. О больших уклонениях от инвариантной меры. - Теория вероятн. и ее примен., 1977, т. 22, в. 1, с. 27-42.

13. Orey S., Pelikan S. Large deviation principles for stationary processes. - Ann. Probab., 1988, v. 16, № 4, p. 1481-1495.

14. Veretennikov A. Yu. On large deviations for ergodic process empírical measures. Adv. Soviet Math., 1992, v. 12, p. 125-133.

15. Wu. Grandes déviation pour les processus de Markov essentiellement irréductibles. I. Temps discret. - C. R. Acad. Sci. Paris, 1991, v. 312, № 1, p. 609-614.

16. Jain N. C. Large deviation lower bounds for additive functionals of Markov processes. - Ann. Probab., 1990, v. 18, № 3, p. 1071-1098.

17. Биллиягсли П. Сходимость вероятностных мер. М.: Наука, 1977, 352 с.

18. Скороход A. B. Асимптотические методы теории стохастических дифференциальных уравнений. Киев: Наукова думка, 1987, 328 с. 